\title{
Interference Effects in T-MOKE Spectra of Fe Thin Films at the $3 p$ Edges - Theory and Experiment
}

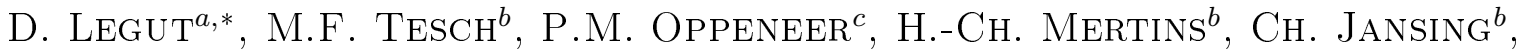 \\ M. Gilbert ${ }^{b}$, A. GaupP ${ }^{b}$, D.E. Bürgler ${ }^{d}$ And C.M. Schneider ${ }^{d, e}$ \\ ${ }^{a}$ VSB-Technical University of Ostrava, 17.listopadu 15, CZ-708 33 Ostrava, Czech Republic \\ ${ }^{b}$ University of Applied Sciences Münster, Stegerwaldstraße 39, D-48565 Steinfurt, Germany \\ ${ }^{c}$ Department of Physics and Astronomy, Uppsala University, P. O. Box 516, S-751 20 Uppsala, Sweden \\ ${ }^{d}$ Forschungszentrum Jülich GmbH, D-52425 Jülich, Germany \\ ${ }^{e}$ Fakultät für Physik and Center for Nanointegration Duisburg-Essen (CeNIDE), Universität Duisburg-Essen, \\ D-47048 Duisburg, Germany
}

\begin{abstract}
We present combined first-principle calculations and experimental results of the transversal magneto-optical Kerr effect (T-MOKE) of thin Fe films across the $3 p$ edges using linearly polarized synchrotron radiation. We show that the experimental T-MOKE spectra at the $3 p$ edges of Fe exhibit clear signals that are strongly influenced by interference effects. Ab initio calculated T-MOKE asymmetry spectra confirm the importance of interference effects. The comparison of experimental with calculated spectra reveals some differences that we attribute to metal/metal interface roughness that is not taken into account in the calculations.
\end{abstract}

DOI: 10.12693/APhysPolA.127.466

PACS: 75.30.Gw, 78.20.Ls, 78.70.Ck, 74.20.Pq

\section{Introduction}

The transversal magneto-optical Kerr effect ( $\mathrm{T}$ MOKE) in the soft $\mathrm{x}$-ray regime is a powerful tool to investigate ferromagnetic materials. It detects a material's optical response that is linear in the magnetization and hence strong [1], ensuring clear magneto-optical spectra. The investigation of the T-MOKE at the $2 p$ edges using hard $\mathrm{x}$-rays is well established, but at $3 p$ edges using extreme ultra violet radiation it is less investigated $[2,3]$. The influence of optical effects like interference in these two energy regimes is different and therefore of great importance for the understanding of measured magneto-optical spectra. In this paper we present energy dependent T-MOKE measurements across the $3 p$ edges of crystalline body-centered cubic (bcc) Fe films with varying thickness and utilize first-principles calculations to obtain insight into the thickness dependence and interference effects in the T-MOKE spectra.

\section{Methodology}

The experimental setup for the T-MOKE measurements is shown in Fig. 1. The linear p-polarized light is reflected from the sample that is magnetized within the surface plane and perpendicular to the plane of incidence. The reflectance of the sample differs for the two antiparallel directions of the magnetization $\left(M_{+}\right.$and $\left.M_{-}\right)$. The T-MOKE asymmetry signal,

$$
A_{\mathrm{T}-\mathrm{MOKE}}=\frac{R_{p p}\left(M_{+}\right)-R_{p p}\left(M_{-}\right)}{R_{p p}\left(M_{+}\right)+R_{p p}\left(M_{-}\right)}
$$

is deduced from two measurements.

The here pre-

\footnotetext{
*corresponding author; e-mail: dominik. legut@vsb.cz
}

sented measurements were performed with the BESSYpolarimeter [4] using linear polarized light of the undulator beamline U125-2-SGM2 with a spectral resolution across the Fe $3 p$ edge (near $54 \mathrm{eV}$ ) of $E / \Delta E=3000$. The angle of incidence, where normal $\theta=0^{\circ}$, was set to $\theta_{i}=50^{\circ}$. For comparison with $a b$ initio calculated T-MOKE spectra the electronic structure of bulk bcc Fe $(a=2.87 \AA)$, was calculated with the WIEN2k code [5] using the local spin density approximation (LSDA) [6] for the exchange-correlation term and including the spinorbit interaction. Details of the calculations are given in Ref. [7]. The absorptive permittivity tensor elements $\epsilon_{i j}(\omega)$ were computed from the linear response expressions, after which the full complex permittivity tensor was obtained by Kramers-Kronig transformation [8]. The method to include roughness for the first $\mathrm{Au} / \mathrm{Fe}$ interface and the optical approach to describe multilayers are given in detail in Ref. [7]. The samples consists of a GaAs substrate covered with a $1 \mathrm{~nm}$ Fe nucleation layer and a $150 \mathrm{~nm} \mathrm{Ag}(001)$ buffer layer [9]. On top of the $\mathrm{Ag}$ the Fe layer is deposited and capped with a $3 \mathrm{~nm}$ Au layer to prevent oxidation. The crystal structure was confirmed by LEED. The sample surface rms roughness was measured by interference microscopy with white visible light giving $3.5 \pm 0.4 \mathrm{~nm}$. The samples are mounted so that the sample edge, i.e. the easy axis, was parallel to the plane of incidence. To investigate the influence of interference effects, four sample were studied, with a thickness of the Fe layer of $5 \mathrm{~nm}, 10 \mathrm{~nm}, 20, \mathrm{~nm}$, and $30 \mathrm{~nm}$. All samples were magnetically saturated. For thick, volume like samples this was demonstrated for a sample $(d=50 \mathrm{~nm})$ showing saturation at $2.5 \mathrm{mT}$ for the easy axis and $80 \mathrm{mT}$ for the medium hard axis. For the in-plane magnetization the effect of the epitaxial strain on the structure and 
hence on MO spectra could not be observed, which is in accordance to results of other groups [10]. Therefore a perfect bcc structure was used for our first-principle calculations.

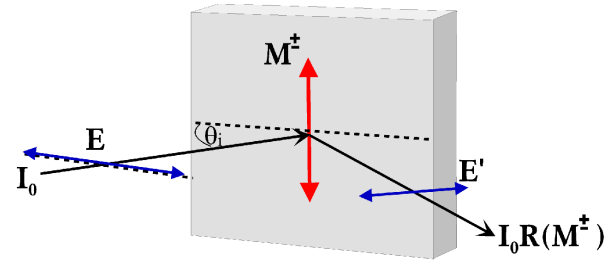

Fig. 1. The experimental setup to measure the TMOKE spectra.

\section{Results}

Reflectivity spectra have been measured in T-MOKE geometry on bcc Fe films with positive and negative applied magnetic field, as sketched in Fig. 1. To discuss the principal physics the averaged spectra for both directions are shown in Fig. 2 (top) together with the resulting T-MOKE asymmetry spectra (bottom). The result of different samples with $5,10,20$, and $30 \mathrm{~nm}$ thicknesses across the $3 p$ edges for the incidence angle $\theta=50^{\circ}$ measured from the surface plane and for the $p$-polarization are given. The positions of the $3 p j_{z}$ states (vertical bars in Fig. 2) are obtained by our $a b$ initio calculations. In addition to the spectral features due to atomic transitions, interference effects occur leading to a modulation of the spectra which will be discussed in the following. These modulations stem from the fact that the incidence photon wave length is about $23 \mathrm{~nm}$, i.e. comparable with sample thicknesses. The reflectivity spectra show two main structures located around $52 \mathrm{eV}$ and around 55-57 $\mathrm{eV}$. These are in rough accordance with the $a b$ initio calculated position of the $3 p$ levels that are splitted in six $j_{z}$ states. Due to the interference effects the atomic spectral features are superimposed by minima and maxima of the reflectivity across the $3 p$ edge for various thicknesses. The 5 and $20 \mathrm{~nm}$ reflectivity spectra are similar, having a reflectivity minimum followed by maximum, whereas the spectral shape for the 10 and $30 \mathrm{~nm}$ samples has two maxima across the $3 p$ edge. The T-MOKE asymmetry shows a similar behaviour. Here, the $5 \mathrm{~nm}$ sample exhibits a maximum for the pre-edge region and then has the lowest minimum of T-MOKE asymmetry signal $(-18.5 \%)$ at $54 \mathrm{eV}$. All other thick samples exhibit similar T-MOKE asymmetry spectra just without the pre-edge maximum. The signal for the T-MOKE asymmetry for the 20 and $30 \mathrm{~nm}$ thick samples have rather similar spectral shapes in contrast to the averaged reflectivity (compare spectral features of top and bottom panels of Fig. 2) and at the same time the signal decreases due to the higher reflectivity (T-MOKE asymmetry normalization) with thickness.

The interference in the reflectance spectra can be explained as follows. The reflected light is decomposed into three rays: the first is reflected at the top vacuum/Au interface, the second at the bottom $\mathrm{Au} / \mathrm{Fe}$ interface, and

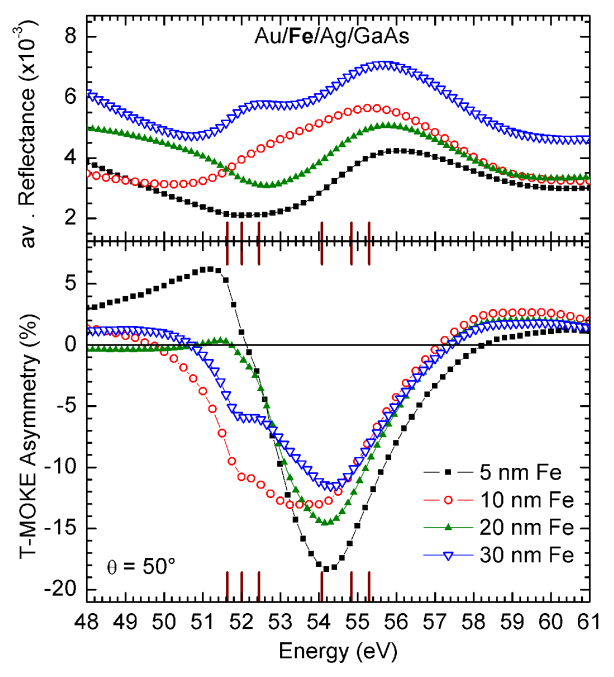

Fig. 2. Measured averaged reflectivity (top) and TMOKE asymmetry spectra (bottom) for incidence angle $\theta=50^{\circ}$ from the surface plane across the $3 p$ edges for the 5, 10, 20 and $30 \mathrm{~nm}$ thick bcc Fe films. The short vertical lines indicate the $3 p j_{z}$ levels as obtained from $a b$ initio calculations.

the third at the bottom $\mathrm{Fe} / \mathrm{Ag}$ interface. The first and second rays are independent of the Fe thickness. The second and the third ray contain information about the $\mathrm{Fe}$ layer and will be of importance for the spectra. For the $d=5 \mathrm{~nm}$ sample mainly destructive interference of the second and the third ray occurs. This results from the phase difference of about $\lambda$ due to the pathway of the light traveling twice through the $5 / \sin \left(40^{\circ}\right)$ nm layer thickness plus the phase shift of $\lambda / 2$ due to reflection at the $\mathrm{Au} / \mathrm{Fe}$ interface. For the other samples with $d=10,20,30 \mathrm{~nm}$ of Fe, interference is less pronounced due to the strong absorption of the third ray when passing through the 10, 20 and $30 \mathrm{~nm}$ thick Fe film. For the thin sample $(d=5 \mathrm{~nm})$ the third ray can travel through the Fe layer without significant absorption before being reflected at the $\mathrm{Fe} / \mathrm{Ag}$ interface. The strength of absorption results from the penetration depth of the light, which varies from $35 \mathrm{~nm}$ below the Fe $3 p$ edge to $9 \mathrm{~nm}$ at and above the $3 p$ absorption edge.

In Fig. 3 the $a b$ initio calculations of the averaged reflectivity and T-MOKE asymmetry are shown. In contrast to the measured reflectivity, the calculated reflectivity of various Fe thicknesses differ only in the pre-edge energy range in particular for the 5 and $10 \mathrm{~nm}$ samples, where the constructive and destructive interference effects are the largest. The calculations show strongest dependence on the film thickness for the pre-edge region. Here the penetration depth through the Fe layer is large resulting in stronger interference effects. Above the absorption edge, the rays traveling through the Fe layer are strongly damped and thus no thickness dependence is observed. This behavior can also be found in the resulting T-MOKE spectra, where for the 20 and $30 \mathrm{~nm}$ samples the spectra are of similar magnitude, while the largest 


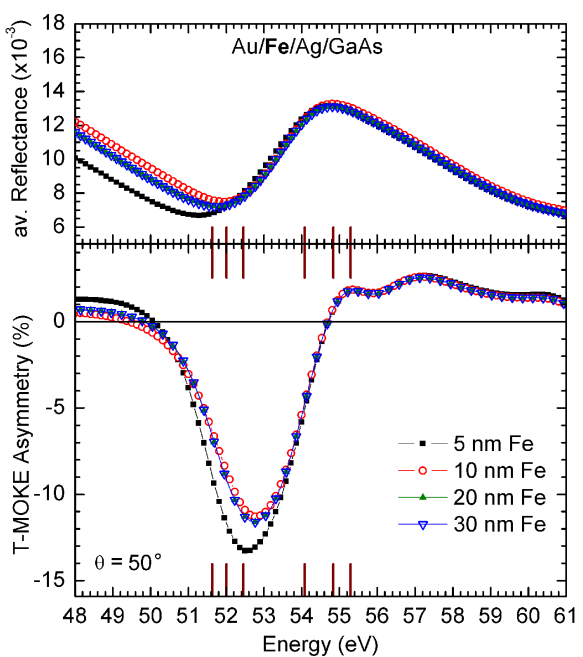

Fig. 3. The same as in Fig. 2, but showing the $a b$ initio calculated T-MOKE spectra.

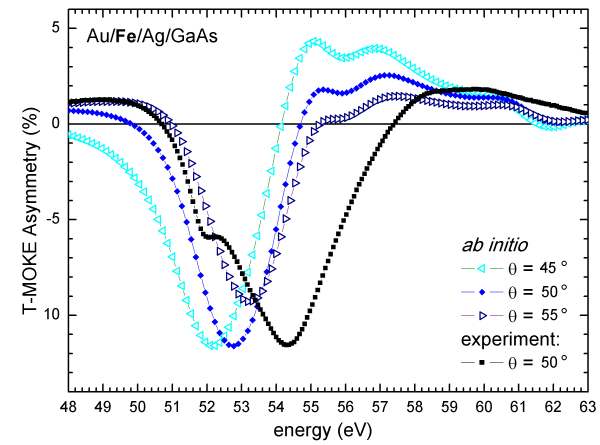

Fig. 4. Calculated T-MOKE asymmetry across the $3 p$ edge of $30 \mathrm{~nm}$ bcc $\mathrm{Fe}$ as a function of grazing angle $\theta$.

difference occurs between the 5 and $10 \mathrm{~nm}$ spectra. In contrast to the experimentally measured spectra the calculated ones (i) are of a smaller width, that might be due to interface roughness, (ii) do not show a positive lobe for $5 \mathrm{~nm}$ sample prior to the $3 p$ edge, and (iii) are shifted to lower energy, i.e. they suffer from the under-binding of the LSDA approximation within the single particle DFT theory.

The calculated T-MOKE asymmetry in the Brewster angle region around $45^{\circ}$ as a function of the incidence angle for the $30 \mathrm{~nm}$ bcc Fe is presented in Fig. 4. The shift of the T-MOKE asymmetry minimum to lower energies is due to the averaged reflectivity that decreases with angle of incidence up to $\theta=50^{\circ}$ (not shown). The measured spectra are much wider and have a shoulder around the energy of $52 \mathrm{eV}$ that does not appear in the $a b$ initio spectra. This must be due the interference effects which are sensitive to the angle of incidence. In contrast, for XMLD spectra [7] in normal incidence theoretical and experimental results are in agreement. Additionally, interface roughness has to be taken into account in future calculations.

\section{Conclusion}

In conclusion, we have measured the T-MOKE spectra across the $3 p$ edge of bcc Fe films. The magnitude of the T-MOKE asymmetry is modified by the film thickness giving rise to the presence of interference effects, as the wavelength is comparable to the film thickness. The firstprinciples calculations are in good accord with the measured T-MOKE asymmetry spectra. The main difference between measured and calculated reflectivity appears to stem from the roughnesses at the $\mathrm{Au} / \mathrm{Fe}$ and $\mathrm{Fe} / \mathrm{Ag}$ interfaces, which at present are not taken into account in our optical code, and therefore further development is needed.

\section{Acknowledgments}

This work has been supported through the Swedish Research Council (VR) and the Swedish National Infrastructure for Computing (SNIC). D.L. acknowledges support by the Grant Agency of the Czech Republic, Project No. 13-30397S, and within the Projects Nos. CZ.1.07/2.3.00/20.0074 and CZ.1.05/1.1.00/02.0070. D.L., M.T., and H.-Ch.M. acknowledges also the Mobility grant 7AMB13DE004 and HZB for technical and financial support. This work acknowledges the COST Action MP1306 EUSpec.

\section{References}

[1] J. Stöhr, H.C. Siegmann, Magnetism - From Fundamentals to Nanoscale Dynamics, Springer, Berlin 2006.

[2] M. Hecker, P.M. Oppeneer, S. Valencia, H.-C. Mertins, C.M. Schneider, J. Electr. Spectr. Related Phenom. 144, 881 (2005).

[3] S. Valencia, A. Gaupp, W. Gudat, H.-C. Mertins, P. M. Oppeneer, D. Abramsohn, C. M. Schneider, New J. Phys. 8, 254 (2006).

[4] F. Schäfers, H.-C. Mertins, A. Gaupp, W. Gudat, M. Mertin, I. Packe, F. Schmolla, S.D. Fonzo, G. Soullié, W. Jark, R. Walker, X.L. Cann, R. Nyholm, M. Eriksson, Appl. Opt. 38, 4074 (1999).

[5] P. Blaha, K. Schwarz, G.K.H. Madsen, D. Kvasnicka, J. Luitz, An Augmented Plane Wave + Local Orbitals Program for Calculating Crystal Properties, Techn. Univ. Wien, Wien 2001.

[6] J.P. Perdew, Y. Wang, Phys. Rev. B 45, 13244 (1992).

[7] M. F. Tesch, D. Legut, H.-C. Mertins, M.C. Gilbert, C. Jansing, J. Hamrle, J. Rusz, P.M. Oppeneer, D.E. Bürgler, C.M. Schneider, A. Gaupp, U. Berges, Phys. Rev. B 89, 140404 (2014).

[8] C. Ambrosch-Draxl, J.O. Sofo, Comp. Phys. Commun. 175, 1 (2006).

[9] D.E. Bürgler, C.M. Schmidt, J.A. Wolf, T.M. Schaub, H.-J. Güntherodt, Surf. Sci. 366, 295 (1996).

[10] F. Nolting, D. Legut, J. Rusz, P. M. Oppeneer, G. Woltersdorf, C.H. Back, Phys. Rev. B 82, 184415 (2010). 